\title{
Reliability and Efficiency at Global Level in Power Systems
}

\author{
A. Jokić ${ }^{1}$, P.P.J. van den Bosch ${ }^{2}$, A. Virag ${ }^{2}$, W.H.A. Hendrix ${ }^{2}$, L. Puglia ${ }^{3}$, W. de Boer ${ }^{4}$, R. Vujanic ${ }^{5}$, F. Nobel ${ }^{6}$ \\ ${ }^{1}$ University of Zagreb, Zagreb, Croatia, ajokic@fsb.hr \\ ${ }^{2}$ Eindhoven University of Technology, Eindhoven, The Netherlands \\ ${ }^{3}$ Institute for Advanced Studies, Lucca, Italy \\ ${ }^{4}$ DNV KEMA Energy \& Sustainability, Arnhem, The Netherlands \\ ${ }^{5}$ Eidgenössische Technische Hochschule Zürich, Switzerland \\ ${ }^{6}$ TenneT, Arnhem, The Netherlands
}

\begin{abstract}
In EU project E-Price it is proposed to attribute reliability to the Transmission System Operator (TSO) and the drive for efficiency to the Balance Responsible Parties (BRP). Two main ideas are proposed. The first idea is to make BRPs themselves responsible for estimating their uncertainty in real time and hedge their imbalance risks, yielding the proposal to introduce dual-sided markets for ancillary services. The second idea is to reduce the conservatism in exploiting the inter-area transmission capacity (ATC), while still guaranteeing sufficient degrees of reliability. This paper explains, elucidates and concludes, based on qualitative arguments and supported by quantitative simulations and calculations, that both proposals are beneficial to improve the stated compromise between reliability and economy.
\end{abstract}

Index Terms-Power system, ancillary services, network constraints, reliability, efficiency.

\section{INTRODUCTION}

Future developments in power systems pose huge challenges for its reliable and economic operation. E-Price (FP7 EU project: Price-based control of Power Systems) focuses on the real-time power imbalance, which arises as a consequence of errors in the prediction of both production and demand. As this (real) power imbalance is expected to increase, both in size and in frequency, present arrangements to cope with this imbalance may become insufficient. They are neither reliable nor economic anymore. This project proposes an advanced ICT and control framework for ancillary services (reserve capacity). This framework allows a more intelligent solution by giving consumers and producers clear, real-time financial incentives to adapt their consumption/production according to the actual needs of the power system. The E-Price framework proposes a two-sided market for ancillary services [4]. This enables BRPs to guarantee sufficient amount of ancillary services when needed to reduce their imbalance costs. The market allows

Project E-Price:Price-based control of Power Systems, supported by EU. FP7 ICT-2009.6.3 for energy efficiency 249096, Info@e-priceproject.eu; p.p.j.v.d.bosch@tue.nl them to make a calculated decision whether it is cheaper to reserve the ancillary services available with own facilities, to buy/sell them on the market or to rely on the, a priori unknown and higher imbalance prices of the TSO. The TSO assesses whether the spatial distribution of reserve capacity in the network can satisfactorily cope with expected classes of disturbances. On the other hand, the E-Price design is based on a distributed control structure, enabled by a fast ICT infrastructure and advanced control theory to reliably and economically deal with ancillary services. Decisions by consumers, producers, power exchanges and TSOs can be taken locally, based on local or national preferences and regulation. Still, the embedded incentives of the proposed framework can guarantee that all these local decisions together contribute to the global EU objectives of the power system: a reliable and efficient electric energy supply.

\section{E-PRICE CONTRIBUTIONS}

In the power system we distinguish two modes of operation: (day) ahead markets and real time. The ahead markets clear the bids of BRPs for energy (EX) and ancillary services (AS), yielding prices and energy volumes for each time period (PTU). The TSO has to cope with the network congestion in an optimal and reliable way. In real time production and consumption will deviate from their predictions and a power imbalance will occur. The TSO calls the available ancillary services (cleared at the AS markets) when needed to control the power balance. With proper incentives in place, BPRs are motivated to cooperate to maintain the system reliability. So, E-Price explores the idea of a dual-sided market for ancillary services to cope with uncertainty. It is based on risk management by BRPs of their own expected imbalances and their supplied/requested AS capacities. A presently existing approach for a BRP to reduce its imbalance risks is to utilize the intraday market (IDM) by contracting energy volumes. As the intraday market clears only a few hours before real-time, the remaining uncertainty 
after closing of ahead markets can be traded on the intraday market, although in some markets the liquidity is very low and, consequently, the uncertainty is quite large. Using AS markets or IDM is different: In the AS markets capacities are reserved and only used when needed, in the IDM volumes are traded, which have to be realized. The realization of these concepts required the development of new algorithms and the integration of those algorithms describing the TSO, BRP and prosumers in both the ahead markets and/or in real-time. For the TSO the network constraints are now explicitly taken into account. For the BRPs the options are realized to bid at the same time on both the EX and the AS markets and to deal, in real-time, with their price-elastic prosumers. The bids for the energy and ancillary service markets are interdependent as they concern the same physical units. As the bids are based on assumptions and suffer from uncertainties, new algorithms have been designed to capture these uncertainties both in a deterministic and in a stochastic framework [1]. Based on historical information the behavior of renewables, other BRPs and the TSO (a BRP's environment) is being estimated and used for better predictions for the coming day to improve the BRP's own performance and/or maximize its profits.

Based on the area control error (ACE) the TSO calculates in real time the needed control/balancing power $\Delta p$ and distributes it among the participating BRPs. The distribution factor $\alpha_{i}$ is based on the cleared contributions of the BRPs in the ancillary service markets. A BRP has to optimize in realtime the setpoints for its generating units and its real-time prices for its price-elastic prosumers to meet in each PTU the agreed volumes of the energy market $(E X)$, the agreed volumes of bilateral trade (OTC) and possibly the volumes contracted by the TSO after closing the ahead markets to cope with network constraints (CT: counter trading).

\section{DUAL-SIDED ANCILLARY SERVICE MARKETS}

BRPs have the option to control their own uncertainty with their controllable power sources and/or by exploiting the sensitivity (e.g. for price) of their prosumers. In this section we focus on global level. There are several options, namely:

- SSAS: Single sided AS market, as it exists nowadays in numerous countries, among which in The Netherlands

- DSAS: Dual sided AS market, a novel concept proposed in E-Price.

- Intraday market (IDM)

In this section their characteristics are qualitatively compared.

\section{A. Ancillary services}

A TSO has to guarantee that sufficient reserve capacity is available when needed in real-time. Markets are created to allow bidding by BRPs with their resources. It is the market design that will guarantee that such a market can be successful. The market design has to be such that the markets are transparent, liquid, with minimal set of rules and such that gaming of any party, either TSO or a BRP, is prevented. In other words, the market arrangements and tuneable parameters have to be selected such that the following 2 requirements can be guaranteed (as already formulated in the Dutch ancillary services arrangements): Forward/Ahead market: The risk of bidding is less or equal
than the risk of not-bidding

In real-time: The risk of a requested action is less or equal than the risk of a not-requested action

\section{1) Single-sided $A S$}

With the single-sided AS market (SSAS) approach, BRPs are obliged by law to offer their spare capacities to the TSO. They are free to assign any price on their bid. This bidding process is considered as a market, although a single-side market with one buyer (TSO) and many sellers (BRPs). This bidding ladder is known in advance. It is used in real-time. When needed the TSO will call these reserved capacities. The more capacity is called by the TSO, the higher the price will be. In general, the highest price occurring in a PTU will be price for AS in that PTU. The responsibility for reserving sufficient capacity is placed at the TSO. So, the TSO determines the size, the BRPs the price.

\section{2) Dual-sided markets}

With the dual-sided AS market (DSAS) approach, the BRPs themselves become responsible for their own uncertainties and reserving sufficient capacity to cover for them. It is allowed to rely on the TSO, but financial incentives make it attractive for them to collect on the DSAS the capacities they expect to need. These capacities have to come from other BRPs. Consequently, the DSAS has to be attractive both for the suppliers and for the requestors of AS. It has to be realized that bids (both as supply (S) and as request (R)) are capacities. There is uncertainty whether the supply bids will be activated or not. So, it can be estimated only with some probability how much will be called and needed. Only a posteriori the volumes are known. That makes it a different commodity compared with the day-ahead energy markets. After clearing of the ahead markets, both sizes [MWh] and prices are known.

In contrast with the SSAS, where BRPs are enforced to offer their bids, the arrangements in the DSAS have to be attractive for BRPs to offer AS. If the rewards for a BRPs become only real when the offered capacity is called in real-time, and there is uncertainty whether the reserved and offered capacity will be called at all, there has to be an incentive to stimulate BRPs to bid sufficient amounts of their spare capacity as AS on the DSAS. In E-Price the following arrangements have been proposed to cope with the requirement to motivate both the supplier and the requestor of AS to participate at the DSAS.

Being supplier of AS, a reward is received proportional with the cleared volume $\mathrm{E}_{\mathrm{AS}, \mathrm{i}}(\mathrm{p})[\mathrm{MWh}]$ and the cleared price $\lambda_{A S}(p)[€ / \mathrm{MWh}]$ in PTU p. Being requestor, a fee has to be paid. So, trading at the AS markets yields always a positive (for S) or negative profit (for R). In both markets the sum of the cleared volumes of suppliers and requestors is zero in 
each PTU. The fixed profits $C_{A S f, i}[€]$ of BRP i after clearing the AS markets equals:

$$
C_{A S f, i}=\sum_{p=1}^{24} a * E_{A S, i}(p) * \lambda_{A S}(p) \text { and } \sum_{i=1}^{\# B R P S} E_{A S, i}=0
$$

The coefficient $a>0[-]$ is a market design parameter. Choosing it too small will reduce the incentive of a BRP for bidding to supply AS, choosing it too large will reduce the incentive of a BRP for bidding to request AS, so an optimal value of $a$ exists which yields the largest participation and so the largest cleared capacity, and, in general, more efficiency so lower prices. In the AS market, the size of a PTU is assumed to be 15 minutes of 1 hour. In figure 1 the expected relations between the sizes of the offered capacities of both suppliers and requestors of AS at a DSAS are influenced by the market design parameter $a$. The best value of $a$ is when the cleared or contracted capacity (red dot in figure 1) obtains its maximum size.

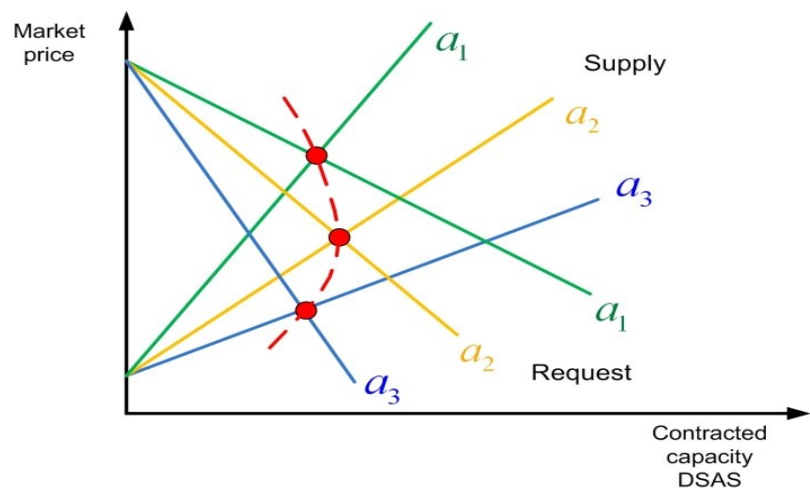

Figure 1. Influence market design parameter a on contracted capacity with $a_{1}<a_{2}<a_{3}$. The red dot symbolises the contracted volume.

\section{B. $\quad$ AS provision in real-time}

A BRP is in imbalance when at the end of a PTU its integrated control error $\mathrm{E}_{\mathrm{imb}, \mathrm{i}}(\mathrm{p})\left\{=\right.$ integral $\left.[\mathrm{P}(\mathrm{k})]-\mathrm{E}_{\text {ref }}(\mathrm{p})\right\}$ over one PTU, starting with value 0 at the start of PTU p deviates from zero. Here, $\mathrm{P}(\mathrm{k})$ is its real power contribution to the grid and $\mathrm{E}_{\text {ref }}(\mathrm{p})$ is its agreed energy contribution in that PTU. When in real-time a global imbalance of the area control error (ACE) exists, the TSO has to buy AS from ASsuppliers to counteract this imbalance. The BRPs causing this imbalance have to pay for their imbalance. The TSO transfers the budgets from the BRPs causing imbalance to the BRPs supplying, on its requests, the needed AS. The following, generic remarks on prices can be made.

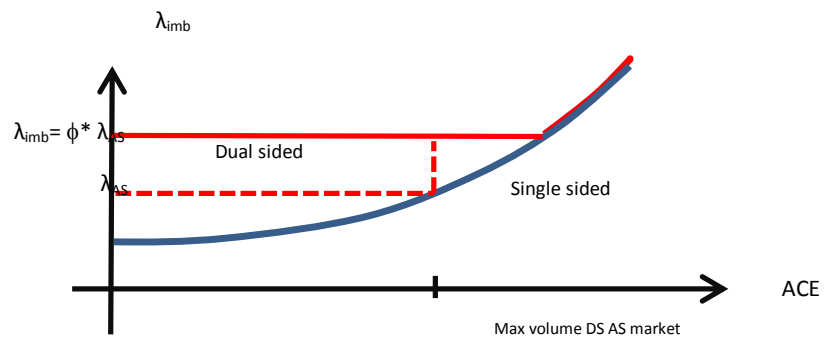

Figure 2. Imbalance price as function of the size of the ACE. Blue curve represents single-sided markets, red double sided markets.
In figure 2, the price at global level for AS $\left(\lambda_{\mathrm{AS}}\right)$ and for being in imbalance $\left(\lambda_{\text {imb }}\right)$ is illustrated for the single (SSAS) and dual sided (DSAS) AS markets.

In the single-sided AS market the TSO calls according to the bid ladder new AS reserves. As the pricing system is marginal pricing, the price will increase when more reserves are needed and are called. In the dual sided market, as long as the TSO can call its reserves cleared at the AS market, the price for being in imbalance will be constant. For BRPs who had cleared their needs at the DSAS, their imbalance is valued with the cleared AS price $\lambda_{\mathrm{AS}}$ (dotted line). For other BRPs being in imbalance the imbalance price $\lambda_{\text {imb }}$ will be used (solid red line). In E-Price we have proposed to make the imbalance price higher than $\lambda_{\mathrm{AS}}$ to make participation in the DSAS more attractive. The parameter $\phi(\phi>1)$ is a market design parameter. Making it too small will not stimulate BRPs to reserve their needed AS capacities at the DSAS, selecting it too large will, unnecessarily, increase the imbalance costs.

Remark: In general the DSAS price is higher than the SSAS price. From this point of view the DSAS is more attractive for a supplier of AS than for a requestor. In next sections, we will show that the indicated prices for AS and for imbalance are the MAXIMUM prices. In general, the prices for AS and imbalance are (considerably) lower for the requestors of AS and imbalance energy. In figure 3 the price to be paid by $\mathrm{BRP}_{\mathrm{i}}$ for $\mathrm{AS}\left(\lambda_{\mathrm{AS}}\right)$ and for being in imbalance $\left(\lambda_{\mathrm{imb}}\right)$ is illustrated for the single and dual sided AS markets.

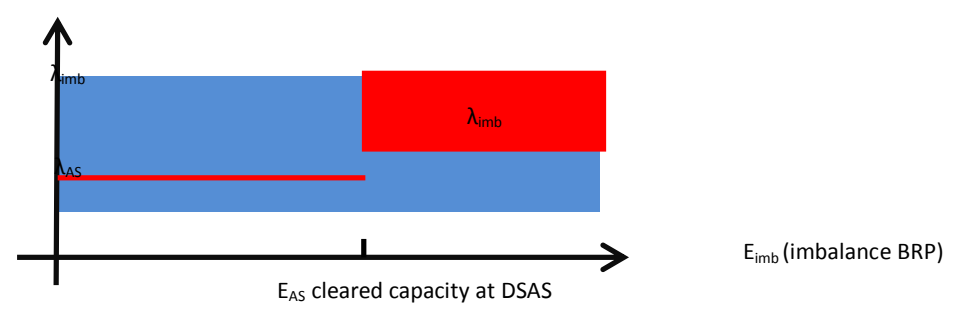

Figure 3: Price when being in imbalance (SSAS in blue, DSAS in red).

SSAS - Single sided market: The imbalance price is a priori unknown and determined a posteriori. It depends fully on the global imbalance of the system and only partly on the own imbalance of the BRP. So, it is uncertain a priori but can be followed with some delay in real time. The uncertainty area is indicated as the large rectangular (blue) area in figure 3.

DSAS - Dual sided market: As long as the imbalance of the $\mathrm{BRP}$ is below its cleared capacity $\mathrm{E}_{\mathrm{AS}}$ at the DSAS, the cleared price $\lambda_{\mathrm{AS}}$ of that market is fixed and known. When the imbalance is larger, the a priori unknown imbalance price $\lambda_{\text {imb }}$ has to be paid. That price is a priori unknown and is indicated as the small rectangular (red) area in figure 3. It is certainly higher than $\lambda_{\mathrm{AS}}$, but its real value depends on the offered bids of the AS-suppliers. Comparing both market outcomes (SSAS and DSAS), it can be concluded that the price to be paid for being in imbalance can be both higher and lower in the DSAS market. The maximum price in the dual sided market is known in advance and is never higher than the own 
bid as long as the own cleared capacity is not being used. Figure 3 illustrates that participation in the DSAS has the characteristic that within its cleared capacity, the price for being in imbalance within the cleared volume is known a priori. In the SSAS arrangement, the imbalance price is, a priori, unknown and determined a posteriori. In the next section it is shown that $\lambda_{\text {imb }}$ is the maximum price. In average it will be lower, depending on the arrangement selected for passive balancing.

\section{Correlation among imbalances of BRP's}

Depending on the source of the uncertainty of the BRP production and/or consumption, there can be no, small, large or complete correlation among the imbalances of the BRPs.

When the sources of uncertainty are fully uncorrelated, the sum of all imbalances will be, on average, zero. The uncorrelated imbalances of the BRPs cancel each other. So, the TSO will call, on average, no AS resources and the costs for imbalance will be low. If BRP_R is the number of BRPs being in imbalance, the correlation $\boldsymbol{\rho}$ can be approximated by

$$
\rho=\frac{\sum_{i}^{B R P_{-} R} E_{i m b, i}}{\sum_{i}^{B R P_{-} R}\left|E_{i m b, i}\right|}
$$

When the sources for uncertainty are fully correlated, so all imbalances have the same sign, the correlation $\boldsymbol{\rho}$ will be 1 (or -1) and the global imbalance will have its maximum value. The sum of the imbalance volumes and the sum of the volumes AS called by the TSO from the BRP_S BRPs supplying $\mathrm{AS}$ on request of $\mathrm{TSO}, \mathrm{E}_{\mathrm{ASs}}$, will be equal. Summarizing:

$$
\sum_{i}^{B R P_{-} S} E_{A S S, i}+\rho \sum_{i}^{B R P_{-} R}\left|E_{\text {imb }, i}\right|=0
$$

This indicates that the correlation $\boldsymbol{\rho}$ among the imbalances matters. The higher the correlation, the more AS will be called. With $\boldsymbol{\rho}=0$, the BRPs causing imbalances cancel the global imbalance completely. No AS has to be called by the TSO.

\section{TSO neutrality}

For a proper regulation it is necessary to guarantee that the TSO, which is responsible for balancing, has no financial interest in executing its role in determining the imbalance price. A consequence will be that the TSO has to be financially neutral and that in each PTU the costs and rewards are being balanced among the BRPs. When considering the correlation $\boldsymbol{\rho}$ among the BRPs, only the situation with $|\boldsymbol{\rho}|=1$ is clear. The BRPs supplying AS will get their reward, while the BRPs being in imbalance have to pay. Both sums are about equal and the TSO will be neutral after the a posteriori accounting transactions.

When $|\boldsymbol{\rho}|<1$, some BRPs being in imbalance will reduce the global imbalance by passively balancing the system. It is assumed that the supplying BRPs still receive the cleared price at the AS market (DSAS). Consequently, the price for $\mathrm{BRP}_{\mathrm{i}}$ for $\mathrm{AS}\left(\lambda_{\mathrm{AS}}\right)$ and the price for being in imbalance $\left(\lambda_{\mathrm{imb}}\right)$ depends, both for the single and dual sided AS markets, on the following arrangements. A BRP being in imbalance, but passively balancing the system (so sign of its imbalance opposite the sign of the global system imbalance), can

1. Pay: any imbalance has to be paid

2. Pay/receive nothing: Pay/receive nothing

3. Receive: passive balancing is rewarded

Owing to the size of correlation $\boldsymbol{\rho}$ both the price for being in active imbalance and the price paid for passively contributing to reducing the imbalance in each PTU have to be reduced. To make these reductions separately visible, in E-Price two different reduction factors have been introduced:

- The prices for paying are reduced with a factor $\gamma(0 \leq \gamma \leq 1)$

- The prices for receiving when passive balancing the system are reduced with a factor $\delta(0 \leq \delta \leq 1)$.

The consequences of this modification are show in table 1 .

When the uncertainties of all BRPs are fully correlated, so $|\boldsymbol{\rho}|$ $=1$, there is no passive balancing. Then $\gamma=1$ and $\delta$ is not relevant. In all other situations $\gamma$ and $\delta$ have to be selected such that the budget the TSO receives equals the budget the TSO has to pay for the called AS energy. In all cases, the solutions will satisfy $0 \leq \delta \leq \gamma \leq 1$. As there is only one constraint (keeping financial transaction in one PTU neutral) and two variables ( $\gamma$ and $\delta$ ), there are many solutions. When comparing the 3 different arrangements, it turns out that arrangement 2 (Nothing) is a special case of arrangement 3 (Receive) by selecting $\delta=0$. Two reasonable choices are $\gamma=$ 1 (highest reward for passive balancing) or $\delta=0$ (lowest AS costs). In both choices, the other variable follows from the financial neutrality requirement in each PTU.

Table 1: Corrected price for being in imbalance, depending on being active contributing to the system imbalance or passive balancing and on whether the own imbalance $\left(E_{i m b}\right)$ in within the cleared capacity $\left(E_{A S}\right)$ at the $A S$ market $\left(E_{i m b} \leq E_{A S}\right)$ or outside $\left(E_{i m b}>E_{A S}\right)$. The sign of the prices is neglected in this survey. When price is printed in bold, the BRP has to pay, when price printed italic the BRP will receive.

\begin{tabular}{|c|c|c|c|c|}
\hline & $\begin{array}{c}\text { Active } \\
\text { imbalance }\end{array}$ & $\begin{array}{c}\text { Active } \\
\text { imbalance }\end{array}$ & $\begin{array}{c}\text { Passive } \\
\text { balancing }\end{array}$ & $\begin{array}{c}\text { Passive } \\
\text { balancing }\end{array}$ \\
\hline Arrangement & $\mathrm{E}_{\text {imb }} \leq \mathrm{E}_{\mathrm{AS}}$ & $\mathrm{E}_{\mathrm{imb}}>\mathrm{E}_{\mathrm{AS}}$ & $\mathrm{E}_{\text {imb }} \leq \mathrm{E}_{\mathrm{AS}}$ & $\mathrm{E}_{\mathrm{imb}}>\mathrm{E}_{\mathrm{AS}}$ \\
\hline 1: Pay & $\gamma^{*} \boldsymbol{\lambda}_{\mathrm{AS}}$ & $\gamma^{*} \lambda_{\text {imb }}$ & $\gamma^{*} \lambda_{\mathrm{AS}}$ & $\gamma^{*} \lambda_{\text {imb }}$ \\
\hline 2: Nothing & $\gamma^{*} \lambda_{\mathrm{AS}}$ & $\gamma^{*} \lambda_{\text {imb }}$ & 0 & 0 \\
\hline 3: Receive & $\gamma^{*} \lambda_{\mathrm{AS}}$ & $\gamma^{*} \lambda_{\text {imb }}$ & $\delta^{*} \lambda_{\text {imb }}$ & $\delta^{*} \lambda_{\text {imb }}$ \\
\hline
\end{tabular}

\section{E. Comparison SSAS and DSAS}

In a DSAS, a requester can limit its liabilities for being in imbalance by bidding for a capacity AS when needed with a maximum price. When the market is cleared, the BRP can be sure that the cleared price is lower than its own costs. Within its cleared volume, the price for being in imbalance is known and lower than it is prepared to pay for. The price to be paid for its risk reduction is a fixed amount of money proportional with the reserved amount of AS capacity. So, a requester of AS can evaluate its costs for risk reduction and, if considered to be profitable, can make its bids on the DSAS. 
Several other remarks can be made to characterize both SSAS and DSAS:

- The BRPs with imbalance have to pay according to their value of $E_{\text {imb }}$ at the end of each PTU. The BRPs that are called by the TSO to deliver AS are paid by the called AS energy during a PTU. Consequently, even in a situation with $|\boldsymbol{\rho}|=1$, the sum of the imbalances $\mathrm{E}_{\text {imb }}$ need not to be equal to the total amount of called AS control energy.

- To maintain the relation between $\lambda_{\mathrm{AS}}$ and $\lambda_{\text {imb }}$ only one $\gamma$ is being used. The added freedom of introducing $\delta$ allows making a compromise between rewarding passive balancing and penalizing being in imbalance. A similar parameter, which regulates the rewards gained by passive balancing, already exists in the Dutch power market. It is called "the incentive component" and can change within a day. Rewarding passive balancing is being paid by BRPs being in active imbalance, not by the BRPs supplying requested AS when called by the TSO. A larger $\delta$ will introduce a larger $\gamma$.

- In the SSAS solution implemented in The Netherlands there is only one price $\lambda_{\text {imb }}$. Financial neutrality of the TSO is approximately achieved by selecting $\gamma=\delta=1$.

- As it is difficult for a BRP to predict whether it will cause or reduce the global imbalance in a PTU, the best arrangement can be defined when, on average, the consequences of causing or reducing the global imbalance cancel each other. Then, too many, sometimes counteracting, control actions at the end of a PTU can be avoided. The market design parameters $\gamma$ and $\delta$ influence the costs of being active in imbalance and the possible rewards of passive balancing. By increasing the rewards (increasing $\delta$ ), also the costs of being in imbalance will increase. Yet, no statement can be made about the best values in a given market environment.

It has been argued that the DSAS is profitable for the supplier of AS and can be profitable for a requester of AS. It depends on the introduced market design parameters $a, \phi, \gamma$ and $\delta$ how much these possible gains can be realized. It is a tradeoff between low AS costs and still sufficient liquidity for the AS markets.

\section{F. Comparison between AS markets and the IDM}

As stated earlier, uncertainty arises from errors in the prediction of production and consumption of tenths of hours ahead. As the ahead markets close in general about 12 hours before starting real-time operation and a day takes 24 hours, predictions have to be made of PTU's from 12 to 36 hours ahead. This large time interval introduces large errors. These errors can be reduced by reducing the time between closing the markets and starting real time. However, many participants in the power system want to have some guarantee in time that their positions can be guaranteed. So, another mechanism to reduce uncertainty is the introduction of the ahead intraday market (IDM), much closer to real time (one hour ahead), but with considerably less volume. It is intended to adjust parties own positions as prediction errors decrease with reduced ahead times.

A real difference between AS markets and the IDM is that at the IDM, just like the day ahead markets (EX, PX), contracts deal with volumes, while at the AS markets the trade is in capacities that can be (partly) called only when needed. A transaction at the IDM can turn out to be counterproductive.

In The Netherlands, the IDM is not largely used. In Germany it is, but there the TSO has to cope with the large uncertainties of the considerable amounts of renewables.

In our separate simulation study [5], based on the assumption that stochastic phenomena of the disturbances can be neglected and that only their statistical properties need to be taken into account, the selection of these market parameters and a comparison with IDM have been made.

A BRP has to assess its probability density function of its net exchange with the grid, as illustrated in figure 4. That function depends on the uncertainty of its own production and consumption.
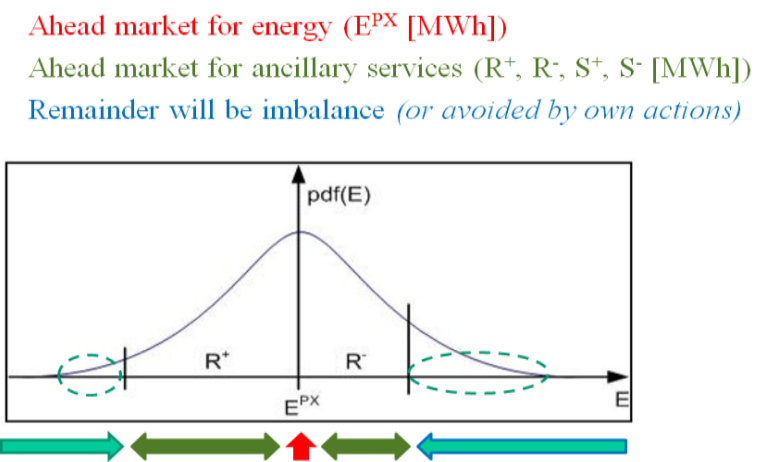

Figure 4. A probability density function of a BRP's expected exchange with the grid in a PTU.

The mean value can be traded at the day ahead market (EX). However, there is a very large probability that there will be an imbalance. By selecting a security level of being in balance with the aid of acquired AS (indicated with $\mathrm{R}^{+}$and $\mathrm{R}^{-}$ in figure 4) at the DSAS, the probability for being in imbalance in real time can be reduced considerably, e.g., to the dashed encircled areas in the figure. However, requesting too much AS has a price, so a compromise has to be made between the costs and the risks. That compromise is at the core of the risk strategy of a BRP [1].

In the following example an indication is given of different approaches for a BRP with a $100 \mathrm{MW}$ wind farm to reduce its uncertainty with realistic measured data from Dutch wind farms [2]. The prediction error 24 hours ahead for wind power has been calculated over 1 year of wind observations and is represented as a probability density function as elucidated in figure 5 .

In the same way the prediction error due to wind forecast for 1 hour ahead has been calculated and represented as a probability density function to mimic the ability to reduce the imbalance by utilizing the IDM. Table 2 illustrates that with a required security level (in table 4 selected as 95, 50 and $0 \%$ ) 
the cleared capacities of AS can be acquired at the DSAS which effectively reduces the remaining imbalance.

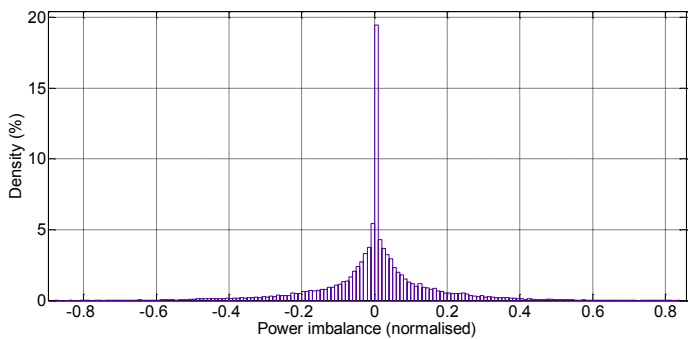

Figure 5: Distribution of the (normalized) prediction error due to (NL) wind power forecast errors 24 hours ahead.

Table 2. Cleared capacities at the DSAS and remaining imbalance depending on selected security level, normalized to the energy traded at the EX $(=100$

$[\%])$.
\begin{tabular}{|l|c|c|c|}
\hline security level [\%] & 95 & 50 & 0 \\
\hline up regulating AS [\%] & 49 & 18 & 0 \\
\hline down regulating AS [\%] & -66 & -19 & 0 \\
\hline remaining imbalance up [\%] & 0.5 & 5.6 & 12.4 \\
\hline remaining imbalance down[\%] & -0.5 & -5.1 & -12.1 \\
\hline
\end{tabular}

With the same approach a comparison has been made among a BRP fully relying on the imbalance system, a BRP acquiring its needed additional volumes at the IDM one hour in advance and a BRP utilizing the DSAS.

Table 3. Net revenue BRP of strategy: no actions to reduce imbalance, using IDM or using DSAS (95\% security level).

\begin{tabular}{|l|c|c|c|}
\hline & $\begin{array}{c}\text { only } \\
\text { imbalance }\end{array}$ & $\begin{array}{c}\text { IDM, } \\
\text { imbalance }\end{array}$ & $\begin{array}{c}\text { DSAS, } \\
\text { imbalance }\end{array}$ \\
\hline revenue EX [M€/year] & 15.61 & 15.61 & 15.61 \\
\hline $\begin{array}{l}\text { all imbalance costs } \\
\text { [M€/year] }\end{array}$ & -0.62 & -0.49 & 0.23 \\
\hline net revenue [M€/year] & 14.99 & 15.12 & 15.84 \\
\hline
\end{tabular}

In Table 3 it is illustrated that in the DSAS approach a BRP can benefit from offering ancillary services and passive balancing. To make a fair comparison, the capacity costs have to be discussed. In The Netherlands the TSO has reserved 300 MW continuously for AS at a cost of about $40 \mathrm{M} €$ per year, so about $135 \mathrm{k} € / \mathrm{MWyear}$. These costs are socialized in the power system, so they are attributed in some way to the producers/consumers.

Table 4 . Net revenue, capacity costs and resulting final revenue depending on strategy of BRP: no actions to reduce imbalance, using IDM or using DSAS (with $95 \%$ security level and $\mathrm{a}=0.1$ )

\begin{tabular}{|l|c|c|c|}
\hline & $\begin{array}{c}\text { only } \\
\text { imbalance }\end{array}$ & $\begin{array}{c}\text { IDM, } \\
\text { imbalance }\end{array}$ & $\begin{array}{c}\text { DSAS, } \\
\text { imbalance }\end{array}$ \\
\hline $\begin{array}{l}\text { revenue at EX } \\
\text { [M€/year] }\end{array}$ & 15.61 & 15.61 & 15.61 \\
\hline $\begin{array}{l}\text { all imbalance costs } \\
{[\mathrm{M} / \text { year] }}\end{array}$ & -0.62 & -0.49 & 0.23 \\
\hline net revenue [M€/year] & 14.99 & 15.12 & 15.84 \\
\hline $\begin{array}{l}\text { capacity costs } \\
\text { [M€/year] }\end{array}$ & -0.33 & -0.33 & -0.63 \\
\hline $\begin{array}{l}\text { final revenue } \\
{[\mathrm{M} / \text { year] }}\end{array}$ & 14.66 & 14.79 & 15.21 \\
\hline final revenue [\%] & 100 & 101 & 104 \\
\hline
\end{tabular}

In the DSAS these capital costs can be assumed to be included in the fixed parameter $a$. In the 2 other cases, they have to be added artificially. There is much freedom to assign these costs and how to select the parameter $a$. The consequences of a reasonable choice (low AS costs and still sufficiently AS liquidity) of these parameters are shown in Table 4.

This example suggests that exploiting the IDM can be profitable. When a DSAS is available, that market is more profitable compared with relying on the IDM and is even more profitable when being unprepared for its own uncertainty and, consequently being enforced to pay the imbalance price.

There are many opportunities to maximize the rewards of the different strategies, all depending on assumptions about prices, probabilities and the reaction of other BRPs and the TSO on the markets and in real time.

The benefits of the proposed DSAS compared with an IDM can be summarized as follows:

- the remaining imbalance of BRPs can be made very small and is controllable by the security level selected or by the confidence interval of uncertainties that are hedged,

- the incorporation of the capacity costs in the DSAS, which is an important distinction with current practice, e.g. intraday markets, is a suitable option compared with present day auction for 1 year,

- the DSAS enables the synergies between a capacity market and the activation of the bids by means of the secondary controller of the TSO. In this approach first all system imbalances are aggregated and only thereafter correcting actions are undertaken, whereas the IDM alone leads to a reduction of individual (BRP) imbalances independent of status of the system imbalance.,

- it is reasonable to state that a DSAS yields more flexibility for BRPs to hedge against uncertainties than only exploiting IDM. The DSAS allows trade in capacities which are reserved, but need not to be taken. The IDM deals with volumes which have to be taken, even if its imbalance was already passive balancing the system.

Therefore the DSAS is a well suited market system to facilitate an increase of renewable generation without additional costs for society for reserving power by the TSO. It allows BRPs to deal efficiently with their uncertainties. Instead of reducing them with own means, cheaper solutions offered by other BRPs at the DSAS can be utilized.

\section{NETWORK CONSTRAINTS AT SYSTEM LEVEL}

With large scale employment of renewable energy (e.g. some 200 Gigawatts of additional renewable sources in EU by 2020), satisfying and "guarding" the global constraints (global power balance, transmission system limits) will become more and more challenging. Also, locations where distributed generation enters the power grid are different when compared to traditional system and future power system will be characterized with new power flow patterns. 
These patterns will be highly uncertain and time varying. It is particularly a challenging task to create efficient solutions on the EU level (the term global then means EU power grid). Expected renewable-energy based grid will change cross border exchanges, will make them more dynamical, and past long-time gathered experiences of controlling the grid will lose some of its value. In future, we will be forced to rely much more on holistic scientific solutions, and much less on experience which will be both scarce and cryptic (unclear how to exploit). It is also increasingly important for market and control solutions to fully use all the existing transmission capacity and to ensure robust operation of the system even in cases of congestion. On a long run, such solutions will send proper signals for optimal investments in the grid, and will accelerate investments in dispersed generation and in new technologies. Interpreting all global power systems problems in terms of global constraints which are to be guarded (in an uncertain time-varying environment) is insightful and a very useful (constructive) way to formulate these problems.

It is important to note that a trade-off between efficiency and reliability is an inherent property of a system with uncertainty.

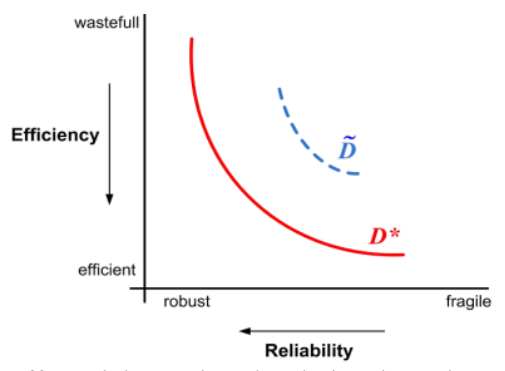

Figure 6: Trade-offs are inherent but also design dependent. A better design pushes the red line to the origin.

Figure 6 illustrates this unavoidable trade-off as larger safety margins to deal with uncertainty will introduce higher costs. Efficiency is the objective function that we like to maximize, while reliability is a set of constraints that limit our feasible set. Then strengthening the constraints (increasing reliability) network constraints not only influence the ahead markets for energy but also influence the supply and need of ancillary services, such that in real-time at any foreseeable disturbance sufficient AS can be made available without violating the network constraints [3]. In real-time, imbalances caused by changes in nodal prosumptions activate units which are contracted at AS markets to change their prosumptions with the goal to restore balance in the system. So, changes in the line power flows are caused by changes in uncontrollable and uncertain nodal prosumptions, and by controlled changes in prosumptions of units that supply AS services. The former we cannot control, but would like to have some knowledge about, while the latter we can control and we have to take care that it is controlled in such a way that line flow limits are not exceeded. The spatial dimension of this problem is obvious as whichever line in the network we observe, how large change of the power flow in this line will be due to the change in some nodal prosumption, heavily depends on where that particular node is.

Example: In E-Price relevant contributions have been made to create pan-European markets for energy and ancillary services [3].

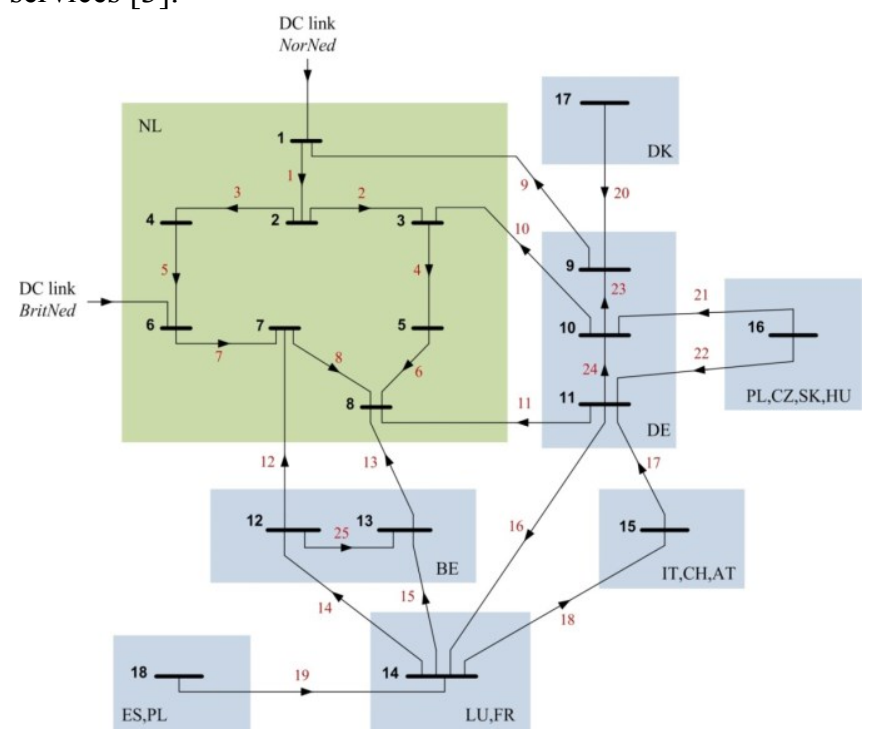

Figure 7. Transmission system topology used in real-time simulations.

Unique solutions have been derived to account for transmission system constraints in AS market clearing. The transmission network topology, as used in the simulations, is presented in Figure 7. The Netherlands, which is in the figure indicated with green area, is modeled by 8 nodes. The neighboring countries are approximated with less nodes. The cross-border connections are with Norway and GB (DC-lines) and $3 \mathrm{AC}$ lines with Germany and $2 \mathrm{AC}$-lines with Belgium. The main goal of this example is to present results of locational (nodal) pricing approach for forward time ancillary services markets. We present the market clearing results for both energy and AS markets, with the aim to illustrate in which way is locational pricing in AS markets similar to the locational pricing in energy market. The simulated outcome of the market clearing prices for energy within the Netherlands are presented in Figure 8, while the corresponding flows in the lines are presented in Figure 9.

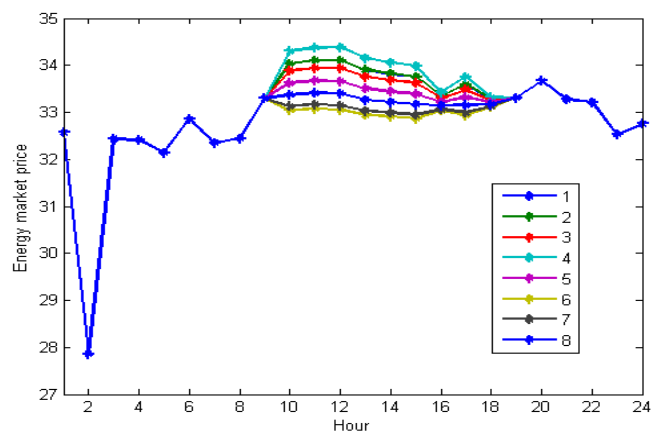

Figure 8. Cleared energy prices (Euro/MWh) over 24 hours. 
When there is no unique price in the system, it must be that at least one tie-line in the transmission network is at its power flow limit (nominal power flow limit). In Figures 8 and 9 this can be observed for the hours 10 to 18 . In that time interval power flow in line 5 is, according to the economically optimal market clearing results, at its nominal power flow limit of $2000 \mathrm{MW}$. During that time interval, there is no unique price in the system (see Figure 8). In case of ancillary services markets, the situation is similar, and described as follows. In Figure ${ }^{1} 10$ we can observe power flows and the "uncertainty power flow tube" in the congestion critical line in the system, that is, in line 5 (see also Figure 9). Note that when the uncertainty tube "touches" the security line flow limit (hour 9, i.e. around $3 \times 10^{4}$ seconds, in Figure 10) we no longer have unique market clearing price for $\mathrm{AS}$ in the system, but each node in general has different AS price.

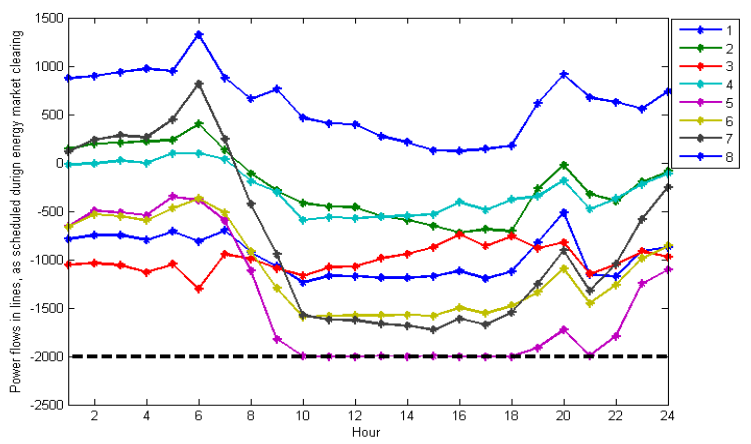

Figure 9. Power flows (MW) in the lines (8 lines within NL) as scheduled during energy market clearing.

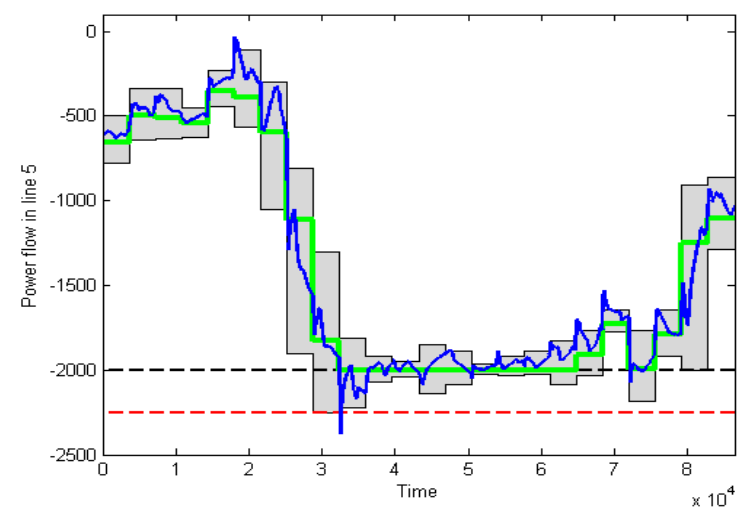

Figure 10. Power flow [MW] in line 5 with nominal value $2000 \mathrm{MW}$.

Green line: tie-line power flow as calculated (predicted) during the energy market clearing

Blue line: real-time realized (simulated) power flow in the tie-line. Deviation of the blue line from the green line originates both from unpredicted changes in load/wind and from activation of AS.

Gray shaded area: is what we will call the uncertainty tube. It is the area for which we can guarantee that the corresponding tie-line power flow will remain in (possibly after a transient excursion outside that area). We guarantee that the AS control action is such that it will not allow power flows to stay outside gray area for longer time periods, or more precisely, that trajectories always converge to within the gray areas.

1 See the legend below the figure for explanations, especially for the definition of the term "uncertainty tube", which plays crucial role in AS markets clearing.
Black dashed line: Nominal line power flow limit (valid and applied in the energy market clearing) of $2000 \mathrm{MW}$.

Red dashed line: Security line power flow limit (valid and applied in the AS markets clearing) of $2250 \mathrm{MW}$.

The analogy with the ahead market clearing (Fig. 8 and 9) is obvious. However, we have to emphasize that the algorithmic solutions for calculating optimal locational AS prices are by no means simple extension of the locational pricing algorithms in the energy market, which are well known and utilized in practice (e.g. in USA). In the AS market clearing, we do not have one singe scalar variable representing power flow in a line, as it is the case in energy market, but we have to deal with the whole set of uncertain power flows (the "uncertainty tube"). Calculation of optimal AS locational prices is based on the robust optimization techniques in combination with Lagrange duality theory. The detailed algorithms have been developed and have presented within the E-Price project [6].

\section{CONCLUSIONS}

E-Price identified the maximization of security of supply and market efficiency as the two main objectives of power system operation. The conflicting nature of these objectives is reflected in the decisions and actions taken by the TSO and the profit-driven BRPs. E-Price proposed two concepts which allow a better compromise than existing arrangements: the dual-sided ancillary service markets (DSAS) and locational prices both for energy and ancillary services in both ahead markets and in real-time. The DSAS design is defined by 4 design parameters which shape its real implementation. It is shown that is has favourable characteristics for BRPs to deal with their trade-off between profits and risk management compared with single-sided AS or intraday market arrangements. In congestion management the tension between reliability and economic efficiency is particularly apparent. Locational prices have shown to be an appropriate tool for solving, in an optimal way, that trade-off. They have been implemented on a close-to reality physical model with 8 separate nodes (copper plates) in The Netherlands. In all realistic simulation studies with considerable uncertainty, still, the network constraints remained satisfied. The proposed algorithms are scalable and can be implemented for the EU power system.

\section{References}

[1] L. Puglia, P. Patrinos, D. Bernardini, A. Bemporad, "Reliability and Efficiency for Market Parties in Power Systems", Proceedings EEM13, Stockholm, 2013.

[2] G.W. Dekker, J. Frunt, W.W. de Boer, M.R. Duvoort, "Case Studies and Results of the E-Price Approach in Power Systems", Proceedings EEM13, Stockholm, 2013.

[3] A. Jokic, Deliverable 2.4 E-Price EU project, 2013.

[4] Bosch, P.P.J. van den, Jokic, A., Frunt, J., Kling, W.L., Nobel, F., Boonekamp, P., Boer, W. de, Hermans, R.M. \& Virag, A. (2011). Price-based control of ancillary services for power balancing. European Transactions on Electrical Power, 21(6), 1889-1901.

[5] W.W. de Boer, M. Duvoort, G. Dekker, "Controlling imbalance risk of wind power with a dynamic spinning reserve margin", 10th International Workshop on Large Scale Integration of Wind Power into Power Systems Aarhus, Denmark, 2011.

A. Jokic, Deliverable 2.2 E-Price EU project, 2013. 